\title{
'Food for thought': The importance of nutrition to patient care and the role of the junior doctor
}

\author{
Authors: Jessica C Frost ${ }^{A_{*}}$ and Alexander ] Baldwin ${ }^{A_{*}}$
}

Good nutrition is an integral component of patient care. Not only does eating correctly provide substantial physical benefits, it also ensures psychological comfort throughout admission. Nevertheless, our formative years as medical students, and now as junior doctors, have shown us that patient nutrition is frequently neglected both in the clinical setting and in the subject matter of our education.

Amid the coronavirus pandemic, this is especially problematic; older, frailer patients, with multiple comorbidities and higher rates of malnutrition, are faring much worse with the virus. Combined with the fact that $40 \%$ of patients admitted to hospital are malnourished to some degree, we are looking at a huge population of potential COVID-19 patients facing a further decline in nutritional status and higher mortality as a result of this, making attention to nutrition more important than ever. As junior doctors, we have a role in the nutritional assessment of and support for our patients by ensuring that all patients are suitably assessed using a scoring tool with the appropriate ensuing actions taken. We must also ensure that our knowledge regarding nutritional assessment and support is adequate and aim to supplement this via additional learning to meet the minimum requirements for our curriculum.

KEYWORDS: nutrition assessment, malnutrition, junior doctors, MUST score, COVID-19

DOI: $10.7861 /$ clinmed.2020-0707

In recent years, also termed by the World Health Organization as the 'United Nations decade of action on nutrition' (2016-2025), collective acknowledgement of the concept of 'food as medicine' has led to nutrition and dietetics rapidly gaining attention both inside and outside of the hospital. ${ }^{1}$ However, despite a growing appreciation for the subject, malnutrition on the wards remains commonplace; as many as $60 \%$ of patients continue to face a decline in nutritional status following admission. ${ }^{2}$ Strict feeding windows, inflexible menus, understaffing and insufficient help for those unable to feed themselves all contribute to the caloric and nutrient deficiencies that patients can face. This is compounded

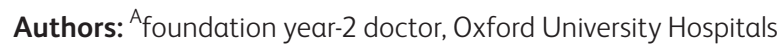
NHS Foundation Trust, Oxford, UK; * joint first authors by the psychological impact of admission, which often results in reduced appetite, inadequate absorption of food, difficulties swallowing, or worsening cognition and confusion, not to mention the physiological effects of illness itself. ${ }^{3}$

The following cases, drawn from personal experience on an acute medical unit, reflect commonly encountered scenarios for the junior doctor and highlight the dynamic nature of nutritional status, and why continual re-assessment is required to prevent its deterioration.

\section{Case 1}

> 60-year-old patient with dysphagia.

$>$ Admitted with an oesophageal food bolus obstruction.

> Kept 'nil by mouth' (NBM) overnight, with plans for oesophagogastroduodenoscopy (OGD) the next morning.

$>$ Unable to fully remove the food bolus with OGD, so remained NBM with a plan to re-scope later that afternoon under general anaesthesia.

> Unfortunately, this was delayed by another day.

> After repeat OGD, at which point the patient had been NBM for more than 48 hours, she was required to remain NBM until Speech and Language Therapy (SALT) assessment.

\section{Case 2}

$>$ Elderly patient with cognitive impairment.

$>$ Admitted delirious with aspiration pneumonia.

> Body mass index (BMI) of $17.5 \mathrm{~kg} / \mathrm{m}^{2}$.

$>$ Kept NBM until SALT assessment.

> No SALT input over the weekend, so remained NBM until the following Monday with no plan in place for their nutrition.

> Patient was difficult to cannulate and pulled out all intravenous access.

> Patient was also not a suitable candidate for nasogastric (NG) feeding.

\section{Case 3}

> Long stay elderly patient on geriatric ward, medically fit for discharge (MFFD), awaiting package of care.

$>$ Thin and wasted with dry scaly skin.

$>$ Nurses noted that the patient had not been finishing meals.

> BMI $18 \mathrm{~kg} / \mathrm{m}^{2}$. 
> No Malnutrition Universal Screening Tool (MUST) score documented.

> Not on oral nutrition supplements, no use of food charts and no referral to dietetics.

\section{Case 4}

> Middle-aged patient with high BMI, hypertension and type 2 diabetes.

$>$ Admitted with COVID-19.

$>$ Due to the illness, they had not eaten for the previous few days.

> Following admission, the patient became increasingly unwell. Their oxygen requirement increased, and they were subsequently admitted to the intensive care unit for intubation and ventilation.

> Subsequent nutritional requirements were met via NG tube.

As illustrated by cases 1 and 2, it is not uncommon for patients who are already nutritionally compromised to experience repeated periods of fasting secondary to procedural delays. These patients need frequent assessment of nutritional status, as well as discussion about alternative methods of hydration, nutrition and medication, and a clear NBM care plan, which should be specific about artificial hydration and electrolyte requirements. ${ }^{3}$ If the patient in question is not suitable for NG feeding, a decision about 'at risk' feeding needs to be made. In scenarios similar to case 2 , where the patient suffers a terminal event in an end-stage disease, it is also important to consider whether palliation may be more appropriate. It is also important to bear in mind that reintroduction of food is not without risk; any patient with a BMI of $<18.5 \mathrm{~kg} / \mathrm{m}^{2}$ and little to no intake for $>5$ days, as in cases 2 and 3 , are in danger of potentially fatal metabolic shifts in fluid and electrolytes on refeeding. ${ }^{4}$ As junior doctors, we need to do what we can to minimise excessive fasting on the ward and ensure that the nutrition team are contacted for support early, especially if NG feeding is required. Enquiry into pre-hospital intake of food is required to further assess this refeeding and malnutrition risk. For patients at high risk of refeeding syndrome, calorie intake should be cautiously increased as advised by the dietetic team, with daily measurement of potassium, phosphate and magnesium levels. Thiamine replacement is also essential. ${ }^{4}$

Case 3 also highlights the importance of nutritionally screening patients on admission, and weekly thereafter, using a validated tool to ensure that any nutritional decline is noticed and appropriately actioned. Just because a patient is deemed 'MFFD' does not mean nutrition should be overlooked. This patient would have a MUST score of at least 2, which puts them in the high-risk category and mandates further action (for example, through prescription of oral nutrition supplements or implementation of a food chart). If a patient has a MUST score of 4 or a score that is increasing, if weight drops by $1 \mathrm{~kg} /$ week, or if intake remains minimal on the ward, a full dietetic assessment should be sought.

Case 4 demonstrates the need for additional nutritional support for COVID-19 patients to help reduce morbidity and mortality associated with the virus, which has been highlighted by multiple nutrition societies worldwide including the European Society for Clinical Nutrition and Metabolism (ESPEN). ${ }^{5}$ The patient in question has a calculated MUST score of 3 due to acute illness and lack of nutritional intake, and warrants dietitian input for consideration of enteral (and potentially parenteral) nutrition therapy. The take home message here is that malnutrition does not discriminate; younger or obese patients, and those with an acute illness, can also be at risk of malnutrition.

In the midst of the coronavirus pandemic, attention to nutrition has become more important than ever. We know that older, frailer patients, with multiple comorbidities and higher rates of malnutrition, are faring much worse with the illness. ${ }^{5}$ Unfortunately, the majority of patients requiring admission with COVID-19 fit into this category, and have pre-existing comorbidities, such as diabetes and cardiovascular disease, both of which are associated with a higher risk of malnutrition., ${ }^{3,6}$ The infection itself is also known to cause a massive reduction in food intake as a result of virus-related anorexia, diarrhoea and vomiting. Combined with the fact that $40 \%$ of patients admitted to hospital are already malnourished to some degree, we are looking at a huge population of potential COVID-19 patients facing a further decline in nutritional status and higher mortality as a result of this. ${ }^{2}$

The COVID-19 pandemic has also imposed additional barriers to the optimisation of patient nutrition. Increased caseloads, redeployment, unfamiliar rules and regimens, remote working and the challenges of personal protective equipment have meant that nutritional screening and assessment has not been a top priority on the wards. ${ }^{6}$ So, how can we, as junior doctors, help to alleviate this nutritional burden on our NHS, during COVID-19 times and going forward?

Paying attention to our patients is the key. Although measurement of water and fat-soluble vitamins can demonstrate specific deficiencies and malabsorption, there is ultimately no single biochemical investigation that can provide concrete evidence of a patient's nutritional state. Medical professionals can become too reliant on albumin, despite the evidence proving that this negative acute-phase protein is affected by multiple factors such as catabolism, sepsis, chronic inflammation, haemodilution, decreased hepatic synthesis or increased excretion, thus rendering it redundant as a marker of nutritional status. ${ }^{4}$

A thorough history (specifically eliciting evidence of weight loss, decreased food intake, reduced appetite or dysphagia) can be helpful. It is also important to note how the patient appears physically. Are they particularly frail and cachectic, obese or oedematous? Careful fluid balance is critical, given that fluid overload or loss can have a confounding effect on measured weight. ${ }^{4}$ Shameer Mehta, in her recent paper on nutritional status and COVID-19, describes how even a single question ('Can the patient finish their meals?') can form the basis of effective nutritional screening. ${ }^{7}$ Making simple observations is something everyone is capable of, regardless of profession or grade. Electronic ward round entries, involving automatically generated prompts, can be programmed to subsequently generate a pre-defined plan for patients; depending on individual risk, resulting actions include repeating the screening 5 days later, initiating volunteerassisted feeding, prescribing oral nutritional supplement drinks or referring to a dietitian.

Ultimately, measurement of weight is essential and usually obtainable. In extremely sick and bedbound patients, where use of a hoist is not feasible, mid-upper arm circumference (MUAC) can be a valuable indicator of malnutrition. Not only can it provide an estimate of BMI, it can also be used to estimate weight change over a period of time. For example, if MUAC changes by $10 \%$, then it is a good estimation that weight has also changed by $10 \%$ or more. ${ }^{8}$ Given that MUST scores are our most valuable resource for identifying malnourished patients, we should start looking to technologise these too. The ability to document patient weight, height and BMI on mobile devices would enable nutritional care 
to become an integrated part of daily practice on the wards, facilitating ward round routine.

The rate of completion of MUST is also an ideal subject for a junior doctor to audit and implement meaningful change via education and intervention. ${ }^{9}$ Our own local data demonstrated a lack of consistency in the completion of nutritional screening, and evidence suggests this is the case across a significant proportion of NHS trusts. ${ }^{10-12}$ One key issue was that the majority of patients who were too unwell, or unable to be weighed, were not being scored, despite the fact that a subjective score based on clinical judgement would be completely appropriate for these cases and definitely better than no score at all!

Ultimately, nutritional review by a consultant (including daily assessment of food and fluid balance charts) needs to be integrated into the ward round pro forma so that it becomes as commonplace, and as easy, as daily antibiotic or analgesia checks. Other easy steps we can all take include the appropriate prescription and adjustment of oral nutrition supplements and careful monitoring of hydration status and suitable fluid replacement. Furthermore, the documentation of patient weight on discharge summaries would enable any nutritional decline or concerns to be flagged up to healthcare professionals in the community. By familiarising ourselves with the criteria for dietitian referrals and expediting these when required, we could hopefully prevent an even greater nutritional decline among those most vulnerable. ${ }^{13}$ Regardless of specialty, there are openings for all doctors (from foundation doctor to consultant) to advocate lifestyle changes to patients or, at the very least, signpost to relevant services or online resources that would undoubtedly have a positive impact on both individual and population health.

Finally, there is undoubtedly a need for more teaching on nutritional assessment, as well as guidance on the prevention of and treatment of malnutrition, and for this to be integrated into the core curriculum of both medical schools and foundation doctors. ${ }^{2,9,14,15}$ For now, the onus remains on ourselves as healthcare professionals to take an interest in the subject and become familiar with the multitude of resources available that cover the basic principles of hospital nutrition so that we can better aid our patients. NHS e-Learning for Healthcare (e-LfH) and British Association for Parenteral and Enteral Nutrition (BAPEN) both offer free nutritional modules for junior doctors that provide simple tools on how to assess nutritional status, fluid management and what to do when caring for patients with nutritional needs, which can also be used towards a foundation trainee's mandatory teaching log.

Ultimately, the battle against malnutrition requires a collective effort; doctors, nurses, medical and nursing students, volunteers and catering staff all have an essential role to play. Nutrition is an underrated and underutilised tool in the hospital arsenal, and one that has the power to prevent a significant number of chronic diseases, as well as the ability to lessen mortality, reduce morbidity and accelerate recovery. It may have taken a pandemic for us to fully appreciate how crucial it is that the nutritional needs of the population are met, especially for those most vulnerable, but Mehta is not alone in viewing this as a unique opportunity for change., ${ }^{7,15}$ COVID-19 is as good a reason as any to start making nutritional care a part of routine practice on the wards, and has highlighted the need for us to shift our emphasis from tangible medical and surgical treatments to a collective culture focusing on the prevention and maintenance of health. However, the challenge remains in us as healthcare professionals, as a team, accepting responsibility and sustaining this for the months and years to come.

\section{References}

1 World Health Organization. Work programme of the United Nations decade of action on nutrition (2016-2025). WHO, 2017. www.who.int/nutrition/decade-of-action/workprogramme2016to2025/en [Accessed 30 March 2020].

2 Elia M; on behalf of British Association for Parenteral and Enteral Nutrition (BAPEN) and the National Institute for Health Research Southampton Biomedical Research Centre. The cost of malnutrition in England and potential cost savings from nutritional interventions. Redditch: BAPEN, 2015. www.bapen.org.uk/pdfs/ economic-report-short.pdf [Accessed 28 July 2020].

3 Leach R, Brotherton A, Stroud M, Thompson R. Nutrition and fluid balance must be taken seriously. BMJ 2013;346:f801.

4 Jones R, Lal S, Shaffer J. Nutrition: tips for the general physician. Clin Med 2012;12:572-5.

5 Barazzoni R, Bischoff S, Breda ] et al. ESPEN expert statements and practical guidance for nutritional management of individuals with SARS-CoV-2 infection. Clin Nutr 2020;39:1631-8.

6 British Diatetic Association. Practical considerations for nutritional management of non-ICU COVID-19 patients in hospital. Birmingham: BDA, 2020 www.bda.uk.com/uploads/ assets/5422cf91-4cd2-4aac-9bc20c2a643f09cd/200512-Practicalconsiderations-for-nutritional-management-of-non-ICU-COVID19-patients-in-hospital-v1.pdf [Accessed 28 July 2020].

7 Mehta S. Nutritional status and COVID-19: an opportunity for lasting change? Clin Med 2020;20:270-3.

8 Todorovic V, Russel C, Elia M; on behalf of the Malnutrition Action Group (MAG), A standing committee of British Association for Parenteral and Enteral Nutrition (BAPEN). The 'MUST' explanatory booklet: A Guide to the 'Malnutrition Universal Screening Tool' ('MUST') for Adults. Redditch: BAPEN, 2003. www.bapen.org.uk/ pdfs/must/must_explan.pdf [Accessed 30 October 2020].

9 Ray S, Laur C, Douglas P et al. Nutrition education and leadership for improved clinical outcomes: training and supporting junior doctors to run 'Nutrition Awareness Weeks' in three NHS hospitals across England. BMC Med Educ 2014;14:109.

10 Norman P. 'MUST do better' - our journey to improving nutrition for everyone - a continuous cyclic, trust wide audit, of NICE Clinical Guideline 32. National Institute for Health and Care Excellence, 2012. https://www.nice.org.uk/sharedlearning/must-do-better-ourjourney-to-improving-nutrition-for-everyone-a-continuous-cyclic-trustwide-audit-of-nice-clinical-guideline-32 [Accessed 28 ]uly 2020].

11 Frank M, Sivagnanaratnam A, Bernstein J. Nutritional assessment in elderly care: a MUST! BMJ Qual Improv Rep 2015;4:u204810. w2031.

12 Russell C, Elia M; on behalf of British Association for Parenteral and Enteral Nutrition (BAPEN) and collaborators. Nutrition screening survey in the UK and Republic of Ireland in 2011. Redditch: BAPEN, 2011. www.bapen.org.uk/pdfs/nsw/nsw-2011-report.pdf [Accessed 28 July 2020].

13 Adamski M, Gibson S, Leech M, Truby H. Are doctors nutritionists? What is the role of doctors in providing nutrition advice? Nutr Bull 2018:43:147-52.

14 Crowley ], Ball L, Hiddink GJ. Nutrition in medical education: a systematic review. Lancet Planet Health 2019;3:379-89.

15 Nowson C. Opportunities for innovation in nutrition education for health professionals. BMJ Nutr Prev Health 2020;3:126-8.

Address for correspondence: Dr Alexander J Baldwin, Department of Oral and Maxillofacial Surgery, John Radcliffe Hospital, Headley Way, Headington, Oxford OX3 9DU, UK. Email: alexbaldwin@doctors.org.uk Twitter: @alex_j_baldwin; @jesscfrost 